\title{
P02-211
}

\section{DEPRESSION, ANXIETY AND HEALTH STATUS IN PATIENTS WITH MULTIPLE SCLEROSIS}

M. Chylova ${ }^{1,2,3}$, J. P. van Dijk ${ }^{3,4}$, J. Rosenberger ${ }^{3}$, I. Nagyova ${ }^{3}$, M. Gavelova ${ }^{5}$, Z. Gdovinova ${ }^{2}$, J. W.Groothoff $^{4}$

11. Department of Psychiatry, ${ }^{2}$ Department of Neurology, Faculty of Medicine, University of PJ Safarik, ${ }^{3}$ Kosice Institute for Society and Health \& Department of Educational Psychology and Health Psychology, Faculty of Arts, University of PJ Safarik, Kosice, Slovak Republic, ${ }^{4}$ Department of Social Medicine, University Medical Center Groningen, University of Groningen, Groningen, The Netherlands, ${ }^{5}$ Department of Neurology, Railways Hospital, Kosice, Slovak Republic

Aims: Multiple sclerosis (MS) is the most common cause of neurological disability in young adults and is frequently accompanied by symptoms of depression and anxiety. The aim of this study was to explore the association of depression and anxiety with health status in younger and older MS patients.

Method: 223 MS patients $(67.3 \%$ female; mean age $38.9 \pm 10.8$ years; mean disease duration $5.8 \pm 5.2$ years) were divided into younger and older age groups (< 45 and $\geq 45$ years). They completed questionnaires focusing on sociodemographic data, depression and anxiety (HADS), and physical and mental health status (SF-36). Functional disability (EDSS) was assessed by a neurologist. To analyse the data, a $U$-test and multiple linear regression analyses were performed.

Results: A model consisting of age, gender, marital status, EDSS, depression and anxiety explained $46.6 \%$ of the variance in physical health status and $60.8 \%$ of the variance in mental health status $(p \leq .001)$. Depression was a significant predictor of physical health status in older MS patients and was associated with mental health status in both age groups ( $\leq \leq 0.001)$. Anxiety was related to worse physical and mental health status in younger MS patients, but not in the older ones.

Conclusion: Depression in MS patients is associated with mental health status and with physical health status only in the older group; anxiety is associated only in younger MS patients with regard to their health status. Psychiatric diagnostics focusing on depression and anxiety might be important for treatment of MS patients in order to contribute to improving a patient's health status. 